\title{
Análise dos fatores que podem contribuir para a ausência ou o preenchimento inadequado da caderneta da criança
}

\author{
Analysis of factors that may contribute to the absence or inadequate filling of the child's \\ book
}
Análisis de factores que pueden contribuir a la ausencia o llenado inadecuado del libro del niño

Maria Rute de Souza Araujo ${ }^{1 *}$, Ana Carolina Marinho Pinheiro ${ }^{1}$, Esther Miranda Caldas ${ }^{1}$, Jucilene Luz Neves ${ }^{1}$, Maria Paula dos Santos Sousa Bulhões Costa ${ }^{1}$, Rogéria de Sousa Rodrigues ${ }^{1}$, Thaís Neves de Souza'.

\section{RESUMO}

Objetivo: Identificar fatores que podem influenciar para ausência ou o preenchimento inadequado da caderneta da criança no que tange ao crescimento e desenvolvimento infantil. Métodos: Trata-se de uma revisão integrativa da literatura, realizada a partir das buscas pelos descritores nas bases de dados SCIELO, LILACS e BDENF em setembro de 2020, os critérios de inclusão estabelecidos dos artigos publicados nos últimos dez anos, estudos em língua portuguesa, disponíveis na íntegra e que abordavam a respeito da temática principal. Resultados: Os fatores que influenciam a ausência ou preenchimento inadequado da caderneta da criança são principalmente a falta de capacitação profissional para o preenchimento adequado, a indisponibilidade da caderneta nas unidades, tempo insuficiente para fazer uma consulta de qualidade e desvalorização do instrumento pelas mães ou responsáveis. Considerações finais: Conforme estudo evidenciou-se a importância do instrumento de vigilância infantil CSC e que com o passar dos anos houve seu intenso aperfeiçoamento, para que assim exercesse seu papel de forma integral a saúde das crianças e norteassem pais, responsáveis e profissionais de saúde para melhores condutas.

Palavras-chave: Saúde da criança, Atenção primária à saúde, Crescimento e desenvolvimento.

\begin{abstract}
Objective: To identify factors that may influence the absence or inadequate filling of the child's handbook regarding child growth and development. Methods: This is an integrative literature review, carried out based on searches by the descriptors in the databases SCIELO, LILACS and BDENF in September 2020, the inclusion criteria established for articles published in the last ten years, studies in Portuguese, available in full and that addressed the main theme. Results: The factors that influence the absence or inadequate filling of the child's handbook are mainly the lack of professional training for proper filling, the unavailability of the handbook in the units, insufficient time to make a quality consultation and devaluation of the instrument by the mothers or guardians. Final considerations: According to a study, the importance of the child surveillance instrument CSC was evidenced and that, over the years, there was an intense improvement, so that it could fully exercise its role in the health of children and guide parents, guardians and health professionals to better conduct.
\end{abstract}

Key words: Child health, Primary health care, Growth and development.

\section{RESUMEN}

Objetivo: Identificar los factores que pueden influir en la ausencia o el llenado inadecuado del manual del niño con respecto al crecimiento y desarrollo infantil. Métodos: Se trata de una revisión integradora de la literatura, realizada a partir de búsquedas por los descriptores en las bases de datos SCIELO, LILACS y BDENF en septiembre de 2020, los criterios de inclusión establecidos para los artículos publicados en los últimos diez años, estudios en portugués, disponibles en su totalidad y que abordó el tema principal. Resultados: Los factores que influyen en la ausencia o llenado inadecuado del manual del niño son

${ }^{1}$ Centro Universitário Metropolitano da Amazônia (UNIFAMAZ), Belém - PA.

*E-mail: mrutearaujo@hotmail.com

SUBMETIDO EM: 2/2021

PUBLICADO EM: 4/2021 
principalmente la falta de formación profesional para el correcto llenado, la indisponibilidad del manual en las unidades, el tiempo insuficiente para realizar una consulta de calidad y la devaluación del instrumento por parte de las madres. o tutores. Consideraciones finales: Según un estudio, se evidenció la importancia del instrumento de vigilancia infantil CSC y que, a lo largo de los años, se produjo una intensa mejora, para que pudiera ejercer plenamente su papel en la salud de los niños y orientar a padres, tutores y profesionales de la salud mejor conducta.

Palabras clave: Salud infantil, Atención primaria de salud, Crecimiento y desarrollo.

\section{INTRODUÇÃO}

Com a implantação dos programas assistenciais à saúde da família, juntamente com Atenção Primária em Saúde (APS), foi possível proporcionar a ampliação do acesso à rede de cuidados voltado para a primeira infância, de acordo com a instituição da Política Nacional de Atenção Integral à Saúde da Criança (PNAISC), baseando-se nos princípios de direitos fundamentais previstos na Constituição Federal e nos princípios do SUS (BRASIL, 2018).

A APS juntamente com a Estratégia Saúde da Família (ESF), buscam promover ações para o bem-estar infantil bem como, comunicação entre os profissionais e a utilização de instrumentos que possibilitem o acompanhamento e vigilância da saúde, com o intuito de buscar a qualidade da assistência demonstrando seu papel fundamental para a eficácia da atenção integral à saúde da criança (SILVA GS, et al., 2018). Nos últimos anos a melhoria da qualidade de saúde das crianças apresentou resultados positivos devido o avanço das políticas públicas e a conquista dos direitos à saúde das crianças, evidenciado pela diminuição do número de mortalidade na infância (BRASIL, 2018).

O crescimento e desenvolvimento infantil tem início desde a concepção até a vida adulta. O crescimento é um processo dinâmico e constante, percebido pela dimensão do tamanho corporal, influenciado por fatores intrínsecos e extrínsecos que indicam a condição de saúde da criança. O desenvolvimento é uma modificação complexa, contínua, flexível, caracterizado pelo desenvolvimento de diferentes habilidades interligadas entre si como o progresso físico, cognitivo e psicossocial (BRASIL, 2012).

No Brasil, a caderneta de saúde da criança (CSC) é utilizada desde 2005, é recomendada pelo Ministério da Saúde (MS) como instrumento de registro de informações da saúde da criança, e visa o acompanhamento do crescimento e desenvolvimento infantil até os 10 anos de idade, estabelece a aproximação dos profissionais de saúde e a família da criança, além de promover a comunicação entre os profissionais nos diferentes serviços de saúde por meio dos registros adequados e periódicos na CSC (AMORIM LP, et al., 2018).

A CSC é um instrumento fundamental para o acompanhamento e desenvolvimento da criança, pois nela contém registros dos dados e informações relevantes para um crescimento e desenvolvimento infantil adequado, para isso deve ter seu preenchimento correto em todos os atendimentos realizados (ABUD SM e GAÍVA MAM, 2015).

Este instrumento propõe o acompanhamento integral da saúde da criança com base na vigilância em saúde, verifica-se que as informações contidas em uma parte são preenchidas pelos responsáveis da criança para nortear as ações voltadas à elas como: orientações gerais sobre saúde e direitos de ambos, em outra parte os profissionais que realizam o atendimento, devem registrar os dados referentes à identificação da criança ainda na maternidade, e outros registros devem ser feitos durante o acompanhamento dessa criança em outros serviços de saúde, estas contemplam a vigilância sobre dados antropométricos em gráfico de crescimento, gráfico de peso, altura e perímetro cefálico de acordo com a idade, amamentação, desmame, alimentação saudável, estimulação e desenvolvimento, tratamentos e suplementação, imunização, e inclusão do guia básico para acompanhar crianças com síndrome de Down e Autismo (ABUD SM e GAíVA MAM, 2015).

A caderneta de saúde da criança é de suma importância se utilizada de forma adequada porque possibilita a interação da família, potencializa o desenvolvimento do cuidado e auxilia na diminuição da 
morbimortalidade infantil identificando situações de riscos e agravos à saúde da criança, sendo fundamental o registro de dados como o peso, visto que são parâmetros para identificar possíveis doenças na fase adulta relacionadas às alterações de crescimento e desenvolvimento ainda intrauterinos, além de contribuir para identificação de possíveis intercorrências, possibilitando a realização de intervenções precoce para evitar o comprometimento da saúde da criança (REICHERT APS, et al., 2016).

Apesar da importância da CSC como instrumento de vigilância no acompanhamento e desenvolvimento infantil, nota-se que a utilização desse instrumento deixa lacunas que prejudicam a avaliação integral dessa criança principalmente no que diz respeito ao crescimento e desenvolvimento, e a falta de registros adequados ou incompletos permitem falhas, as quais impedem que sejam implementadas ações de promoção a saúde voltadas às crianças, a falta de preenchimento adequado dificulta e interfere na assistência dos próprios profissionais, pois é na caderneta que se identifica situações de riscos e agravos à saúde infantil, além, de promover as condutas direcionadas às condições de saúde dessa criança (SILVA FB e GAIVA MAM, 2016).

Diante disto o enfermeiro tem um papel fundamental no preenchimento fidedigno da CSC, visto que é um instrumento de suma importância que tem a capacidade de diminuir os índices de morbimortalidade infantil e proporcionar um avanço significativo na melhoria dos indicadores de saúde. Independentemente de não ser o único profissional a utilizar a CSC tem extrema importância na captação e registro correto dos dados que serão norteadores para um planejamento e implementação do cuidado adequado e o acesso à saúde de forma integral, permitindo a continuidade das ações de prevenção e promoção da saúde infantil (ROSOLEM LH, et al., 2019).

A partir dessa realidade gerou-se uma inquietação de pesquisar sobre a temática, então formulou a questão de pesquisa: Quais os fatores que podem influenciar para ausência ou registros inadequados dos instrumentos de avaliação na caderneta de saúde da criança pelo enfermeiro? Dessa forma, este estudo tem como objetivo identificar os fatores que podem influenciar para a ausência ou o preenchimento da caderneta da criança evidenciado na literatura no período de 2012 a 2019.

\section{MÉTODOS}

Trata-se de uma revisão integrativa da literatura, de caráter descritivo baseadas em estudos publicados no período de 2010 a 2020. A revisão integrativa da literatura é um estudo pelo qual se permite a busca, assim como análise crítica e síntese dos conhecimentos acerca do tema investigado, dessa forma tem como produto final o atual conhecimento a respeito do tema assim como a implementação de uma assistência efetiva no âmbito da saúde, e a identificação de possíveis lacunas as quais conduzem para o desenvolvimento de novas pesquisas (MENDES KDS et al., 2008). A mesma visa analisar a produção científica a respeito do tema investigado, de forma que venha a contribuir para a discussão dos fatores que implicam no preenchimento incorreto na CSD no que se refere ao crescimento e desenvolvimento infantil.

A produção de tal pesquisa se deu através das seguintes etapas: elaboração do tema, bem como a escolha da questão de pesquisa; estabelecimento dos critérios de inclusão e exclusão, com buscas na literatura; coleta dos dados; avaliação crítica dos estudos selecionados; interpretação dos resultados; e síntese dos conhecimentos obtidos.

O levantamento bibliográfico se deu em setembro de 2020 a partir das bases de dados Scientific Eletronic Library (SCIELO), Literatura Latino-Americana e do Caribe em Ciências da Saúde (LILACS), e Bases de Dados de Enfermagem (BDENF) utilizando-se de descritores anteriormente já validados no Descritores em Ciências da Saúde (Decs): "Saúde da criança" "Atenção Primária à Saúde" "Crescimento e desenvolvimento".

Para a busca dos artigos nas bases de dados foram estabelecidos os seguintes critérios de inclusão: artigos publicados nos últimos dez anos (2010-2020), somente estudos em língua portuguesa e disponíveis na integra e que abordavam a respeito da temática principal. Foram excluídos os artigos que não atendiam ao período estabelecido, que não atendiam ao objetivo da pesquisa, artigos em língua estrangeira, além de teses e monografias. 
Após estabelecer os critérios de inclusão e exclusão foram realizadas as pesquisas nas bases de dados com os descritores estabelecidos, as quais seguiram algumas etapas, a primeira etapa consistiu na leitura dos títulos dos artigos, a segunda na leitura dos resumos e a terceira na leitura para a análise crítica bem como avaliação dos estudos anteriormente selecionados. Na primeira e na segunda etapa foram excluídos os artigos que não abordavam sobre o preenchimento da CSD, e que no desfecho não abrangiam sobre os fatores que interferem no preenchimento desta. Na terceira etapa os artigos selecionados para análise foram lidos na íntegra para que fosse verificado a possibilidade de inclusão considerando seus resultados, discussões e os seus desfechos.

Realizou-se de forma descritiva tanto a análise quanto a síntese dos dados extraídos dos artigos para que fosse possível a observação a descrição e a classificação a fim de se garantir a exatidão na verificação das informações.

\section{RESULTADOS}

Após as pesquisas nas bases de dados com os descritores escolhidos foram identificadas no total 81 publicações. Na BDENF identificou-se 21 artigos, na base LILACS foram identificados 33 artigos, já na base de dados SCIELO identificou-se 27 artigos. Em seguida, aplicando-se limites para inclusão, selecionou-se para amostra inicial total de 22 artigos. Posteriormente, ao fim foram realizadas leituras e análises minuciosas na íntegra de cada artigo e separou-se para amostra final 6 estudos, de acordo com o objetivo da pesquisa, sendo eles 2 da BDENF; 2 na LILACS; e 2 na SCIELO.

Posteriormente a análise crítica dos artigos selecionados, foram agrupados 6 artigos para amostra e foi utilizado instrumento adaptado de Ursi (2015), onde foi organizado da seguinte forma: autor/ano/periódico; título; objetivo; resultado (Quadro 1). Após os artigos serem selecionados e investigados, de acordo com seus conteúdos foram divididas duas categorias.

Na primeira categoria, foram adicionados 3 artigos que discorrem sobre tal tema que correspondem aos temas:(1) Evidenciaram-se, neste estudo, fatores que influenciam a precária utilização da CSC, pelos profissionais de saúde, para a vigilância do processo de crescimento e desenvolvimento infantil.(2) Elucidaram-se, com base nos resultados, as falhas nos registros e o devido preenchimento das CSC, mostrando que a maioria dos profissionais não avalia todos os marcos considerados imprescindíveis para a vigilância do C/D da saúde da criança.; (3) A CSC é fundamental e indispensável para o acompanhamento infantil. Percebe-se que o preenchimento da CSC necessita ser aprimorado, para atingir a competência que Ihe é devida.

Os artigos selecionados relatam sobre a carência de capacitação dos profissionais da saúde para realizarem o preenchimento de forma adequada da CSC, pois são vastas as dificuldades enfrentadas por eles desde o tempo da academia. Os artigos tratam a respeito da falta de capacitação do profissional de saúde em preencher a CSC, do não reconhecimento da relevância de um preenchimento adequado e da deficiência de informações em vários requisitos em que ela possui, como gráficos, até mesmo informação pessoal da criança falta de conhecer a devida importância de cada item nela contida entre outros, na qual termina por deixar lacunas imprescindíveis para o acompanhamento e vigilância do crescimento e desenvolvimento infantil.

$\mathrm{Na}$ segunda categoria, foram encontrados 3 estudos compreendendo os seguintes títulos: (1) "Desafios na utilização da caderneta de saúde da criança: entre o real e o ideal"; (2) "Assistência à criança: preenchimento da caderneta de saúde em municípios do semiárido brasileiro"; (3) "Uso e preenchimento da caderneta de saúde da criança com foco no crescimento e desenvolvimento".

Ainda na segunda categoria, 3 artigos abordam a respeito dos fatores que interferem para um preenchimento adequado da CSC, como: a falta de capacitação da equipe de saúde, tempo insuficiente para fazer uma consulta de qualidade devido a um cumprimento de uma agenda, a indisponibilidade da caderneta nas unidades de saúde, a não utilização da CSC por toda a equipe pois todos são responsáveis pelos cuidados com a criança, desvalorização ou desconhecimento da CSC pelas mães ou responsáveis, onde são baseados pelos níveis de escolaridade e situação socioeconômica, atribuindo a importância em suma para vacinação. 
Quadro 1 - Artigos que foram utilizados como amostra selecionados nas bases BDENF, LILACS e SCIELO entre os anos de 2012 a 2019.

\begin{tabular}{|c|c|c|c|c|}
\hline $\begin{array}{l}\text { Autor/ } \\
\text { Ano }\end{array}$ & Periódico & Título & Objetivos do estudo & Resultado \\
\hline $\begin{array}{l}\text { ABREU } \\
\text { TGT, et al., } \\
2012 \text {. }\end{array}$ & $\begin{array}{l}\text { J Manag Prim } \\
\text { Health care, } 3 \\
\text { (2): } 80-3 .\end{array}$ & $\begin{array}{l}\text { Desafios na utilização } \\
\text { da caderneta de saúde } \\
\text { da criança: Entre real e } \\
\text { o ideal. }\end{array}$ & $\begin{array}{l}\text { Objetiva-se então, levantar conteúdos } \\
\text { na literatura acerca dos fatores que } \\
\text { dificultam a utilização adequada da } \\
\text { caderneta de saúde da criança pelos } \\
\text { profissionais de saúde. }\end{array}$ & $\begin{array}{l}\text { Os profissionais enfrentam dificuldades na utilização da CSC: } \\
\text { falta de conhecimento e capacitação para o uso com a } \\
\text { caderneta, tempo insuficiente para dialogar com a família } \\
\text { sobre as informações contidas no instrumento, a falta da CSC } \\
\text { em alguns locais de serviço, a adesão de todos os membros } \\
\text { da equipe na sua utilização. }\end{array}$ \\
\hline $\begin{array}{l}\text { PALOMBO } \\
\text { CNT, et al., } \\
2014 .\end{array}$ & $\begin{array}{l}\text { Rev Esc Enferm } \\
\text { USP, } 48 \text { (Esp): } \\
60-7 .\end{array}$ & $\begin{array}{l}\text { Uso e preenchimento } \\
\text { da caderneta de saúde } \\
\text { da criança com foco no } \\
\text { crescimento e } \\
\text { desenvolvimento }\end{array}$ & $\begin{array}{l}\text { Avaliar uso e preenchimento da } \\
\text { Caderneta de Saúde da Criança (CSC), } \\
\text { especialmente crescimento e } \\
\text { desenvolvimento. }\end{array}$ & $\begin{array}{l}\text { O uso e o preenchimento insatisfatório da CSC reforçam a } \\
\text { necessidade de investimentos na capacitação dos } \\
\text { profissionais e na sensibilização da comunidade para que a } \\
\text { CSC se torne efetivamente um instrumento de promoção da } \\
\text { saúde infantil. }\end{array}$ \\
\hline $\begin{array}{l}\text { COSTA } \\
\text { JSD, et al., } \\
2014 .\end{array}$ & $\begin{array}{lr}\text { Rev. } & \text { Bras. } \\
\text { Saúde } & \text { Matern. } \\
\text { Infant., } & 14 \quad(3): \\
219-227 . & \end{array}$ & $\begin{array}{l}\text { Assistência à criança: } \\
\text { preenchimento da } \\
\text { caderneta de saúde } \\
\text { em municípios do } \\
\text { semiárido brasileiro }\end{array}$ & $\begin{array}{l}\text { Determinar a proporção de crianças } \\
\text { com cadernetas de saúde com } \\
\text { preenchimento adequado e analisar } \\
\text { associações com características } \\
\text { geográficas, socioeconômicas e } \\
\text { biológicas maternas e da criança e com } \\
\text { o percentual de utilização de serviços } \\
\text { de saúde em dois municípios do semi- } \\
\text { árido brasileiro. }\end{array}$ & $\begin{array}{l}\text { Os resultados demonstram baixo percentual de cadernetas de } \\
\text { saúde adequadamente preenchidas. A maior prevalência de } \\
\text { cadernetas com preenchimento inadequado nas mães de } \\
\text { baixa escolaridade denota iniquidade no sistema de saúde } \\
\text { local. O preenchimento adequado é um direito da criança, a } \\
\text { sua inadequação indicou despreparo dos profissionais e dos } \\
\text { pais às necessidades da atenção básica. }\end{array}$ \\
\hline $\begin{array}{l}\text { LIMA LG, et } \\
\text { al., } 2016 .\end{array}$ & $\begin{array}{l}\text { Rev. Brasileira } \\
\text { de ciências da } \\
\text { saúde, } 20 \text { (2): } \\
167-174 .\end{array}$ & $\begin{array}{l}\text { A utilização da } \\
\text { caderneta de saúde da } \\
\text { criança no } \\
\text { acompanhamento } \\
\text { infantil. }\end{array}$ & $\begin{array}{l}\text { O estudo tem por objetivo analisar } \\
\text { artigos da literatura nacional relativo a } \\
\text { utilização da CSC nos serviços de } \\
\text { atendimento materno infantil. }\end{array}$ & $\begin{array}{l}\text { A CSC é fundamental e indispensável para o } \\
\text { acompanhamento infantil. Percebe-se que o preenchimento } \\
\text { da CSC necessita ser aprimorado, para atingir a competência } \\
\text { que lhe é devida. Os melhores percentuais de preenchimento } \\
\text { são nos itens relacionados à identificação da criança, como: } \\
\text { O nome da criança, peso e comprimento. Os percentuais de } \\
\text { preenchimento em relação às curvas de crescimento são } \\
\text { considerados insatisfatório. }\end{array}$ \\
\hline
\end{tabular}




\begin{tabular}{|c|c|c|c|c|}
\hline $\begin{array}{c}\text { Autor/ } \\
\text { Ano }\end{array}$ & Periódico & Título & Objetivos do estudo & Resultado \\
\hline $\begin{array}{l}\text { SILVA TCT, } \\
\text { et al., } 2018 .\end{array}$ & $\begin{array}{l}\text { Rev enferm } \\
\text { UFPE online, } \\
\text { 12(12): } 3445-55 .\end{array}$ & $\begin{array}{l}\text { Caderneta De Saúde } \\
\text { Da Criança: Vigilância } \\
\text { Do Crescimento E } \\
\text { Desenvolvimento } \\
\text { Infantil }\end{array}$ & $\begin{array}{l}\text { Analisar as evidências científicas } \\
\text { quanto à utilização da Caderneta de } \\
\text { Saúde da Criança pelos profissionais } \\
\text { de saúde para a vigilância do } \\
\text { crescimento e desenvolvimento infantil. }\end{array}$ & $\begin{array}{l}\text { Evidenciaram-se, neste estudo, fatores que influenciam a } \\
\text { precária utilização da CSC, pelos profissionais de saúde, para } \\
\text { a vigilância do processo de crescimento e desenvolvimento } \\
\text { infantil, tais como: a ausência e a fragilidade de registros; a } \\
\text { dificuldade dos profissionais perceberem a relevância do } \\
\text { preenchimento; o conhecimento deficiente dos profissionais } \\
\text { sobre a caderneta; a grande demanda no atendimento infantil; } \\
\text { a burocracia do serviço; a fragilidade no processo de } \\
\text { comunicação e no trabalho em equipe; a insuficiência de } \\
\text { orientações às famílias sobre os cuidados à criança, além da } \\
\text { participação da família neste processo. }\end{array}$ \\
\hline $\begin{array}{l}\text { SOUZA } \\
\text { NS, et al., } \\
2019 .\end{array}$ & $\begin{array}{l}\text { Rev enferm } \\
\text { UFPE online, } \\
\text { 13(3):680-9. }\end{array}$ & $\begin{array}{l}\text { Vigilância E Estímulo } \\
\text { Do Crescimento E } \\
\text { Desenvolvimento } \\
\text { Infantil }\end{array}$ & $\begin{array}{l}\text { Avaliar as ações de vigilância e } \\
\text { estímulo, ao crescimento e } \\
\text { desenvolvimento da criança, realizadas } \\
\text { na Atenção Básica de Saúde }\end{array}$ & $\begin{array}{l}\text { Elucidaram-se, com base nos resultados, as falhas nos } \\
\text { registros e o devido preenchimento das CSC, mostrando que } \\
\text { a maioria dos profissionais não avalia todos os marcos } \\
\text { considerados imprescindíveis para a vigilância do C/D da } \\
\text { saúde da criança. Pode-se constatar que o acompanhamento, } \\
\text { seguido da forma preconizada, age como dispositivo de } \\
\text { promoção à saúde, sendo necessário fortalecer e exercitar } \\
\text { ações que objetivem melhorias nos serviços que participaram } \\
\text { da pesquisa. Considera-se que uma boa estrutura e a } \\
\text { disponibilização de equipamentos e insumos são } \\
\text { imprescindíveis para a prestação de uma assistência de } \\
\text { qualidade à criança, e isso possibilita que os profissionais } \\
\text { desenvolvam suas atividades, tendo como base o seguimento } \\
\text { e a implementação das políticas públicas vigentes. }\end{array}$ \\
\hline
\end{tabular}

Fonte: Araujo MRS, et al., 2021. 


\section{DISCUSSÃO}

A CSC traz dados desde a gravidez, a respeito à saúde do recém-nascido como também acompanhamento da saúde bucal da criança, gráficos do crescimento e desenvolvimento, prevenção de acidentes, a mesma também contempla informações a respeito de orientações para uma alimentação saudável, acompanhamento de suplementação de ferro e vitaminas e o calendário vacinal. Sendo assim, quando preenchida corretamente é uma ferramenta de extrema importância para o acompanhamento e promoção de saúde para a criança, permitindo comunicação entre a unidade de saúde e familiares (SALLES IC e TORIYAMA, ATM 2017).

Para Silva TCT, et al. (2018) dentre os fatores citados no estudo do autor foi abordado o despreparo dos profissionais para a utilização da CSC de forma adequada, e até mesmo a falta de conhecer a caderneta, que são bases primordiais para que a mesma seja preenchida corretamente e cumpra seu papel de instrumento de vigilância integral à saúde da criança.

O preenchimento deficiente da CSC só evidencia o precário acompanhamento no que tange ao acompanhamento do crescimento e desenvolvimento de forma integral especialmente na primeira infância, tal fato está intimamente ligado as crianças cujo o cuidador principal não são os pais nem os avós, outros fatores como a ausência do pré-natal bem como as informações advindas deste influenciam para o má preenchimento por parte dos profissionais, principalmente a respeito do desenvolvimento neuropsicomotor (FREITAS JLG, et al.,2019).

Ademais, torna-se um desafio para os gestores dos sistemas de saúde como Estratégias Saúde da Família, unidades básicas de saúde, setores ambulatoriais e hospitalares reinventarem-se para ofertar melhores conhecimentos, cursos, treinamentos a equipe multiprofissional, visto que são frequentes os achados em pesquisas científicas sobre a grande dificuldade dos profissionais em completarem corretamente a CSC, para que assim possa estabelecer se auxílio para modificação desse cenário (SILVA TCT, et al., 2018).

Em seguida, Souza NS, et al. (2019) cita no artigo que a precarização do preenchimento da caderneta de saúde da criança por partes de profissionais de saúde, pois não apresentam capacitação completa, gera consequentemente uma péssima qualidade na assistência, onde abrem lacunas para o preenchimento inadequado, no qual apresenta falhas na complementação de gráficos, curvas de crescimento e desenvolvimento, e medidas antropométricas, dessa forma, fazendo com que tenha uma fragilidade no serviço prestado.

Para Andrade GN, et al. (2014) além do que foi exposto a respeito da falta de capacitação de alguns profissionais, um outro fator que é relatado por eles é a sobrecarga de funções do trabalho, situação que de acordo com os profissionais de saúde acabam fazendo que o tempo de atendimento e adesão à caderneta em sua totalidade fiquem prejudicados.

Abreu TGT, et al. (2012) em seus estudos identificaram como os principais fatores que interferem ou dificultam o preenchimento correto da CSC são: falta de capacitação dos profissionais de saúde pois alegam desconhecer as mudanças e aprimoramentos inclusos a caderneta com o passar dos anos, e relatam não saberem manusear. Assim como, o tempo insuficiente para as consultas são grandes as demandas de atendimento, burocracias para escreverem, alta oferta de pacientes, o que termina por comprometer uma boa assistência.

A carência da caderneta física na instituição de saúde, favorecendo assim o não monitoramento do processo saúde doença destas crianças, bem como o não manuseio da caderneta pelos componentes da equipe o que demonstra a falta de comprometimento de alguns profissionais com a CSC, já que é essencial que essa caderneta seja utilizada pela equipe, para que haja continuidade do fluxo no atendimento, vale ressaltar ainda também a falha de pais ou responsáveis em não dar a importância devida a caderneta, ocorre de perderem, não saberem o seu real objetivo, o que corrobora por não haver a completude nas consultas, até mesmo como eles lidarem com as modificações físicas e psicológicas das crianças no dia a dia (ABREU TGT, et al., 2012). 
Segundo Silva FB e Gaíva MAM (2016), reafirmam em seus estudos e análises que há desafios para os profissionais em preencher CSC, por conta da carência de profissionais nas unidades e burocracias no próprio serviço de saúde. Dessa maneira, para que possa haver mudanças em tal cenário é de extrema relevância que ações sejam tomadas pelas autoridades competentes, pois infelizmente é tendencioso o maior declínio da assistência integral à saúde da criança.

Para Cavalcante JRC, et al. (2018) é importante que os gestores e gerente das instituições estimulem o uso da caderneta pelos profissionais de saúde para a prevenção e a identificação de possíveis agravos ao crescimento infantil, visto que só o acesso pelas mães ou responsáveis não garante a qualidade e a integralidade dos serviços de saúde voltado à criança, o uso correto desta se faz um indicativo importante no que diz respeito ao envolvimento dos profissionais de saúde bem como evidencia o vínculo por parte da família com os serviços de saúde, dessa forma dar-se a importância de ações educativas que proporcione as famílias maiores conhecimentos a respeito da caderneta.

Além disso, de acordo com, Soares JSD, et al. (2014) foram identificadas dificuldades sobre o não preenchimento da caderneta de saúde da criança bem como o despreparo dos profissionais sobre o manejo da caderneta, contendo lacunas de informações sobre peso, IMC, medidas antropométricas e curvas, pois há uma falta de profissionais nas unidades de saúde, fazendo com que sobrecarregue o serviço apenas para uma minoria de profissionais e consequentemente, fazendo com que haja um preenchimento inadequado da CSC.

Ademais, um outro fator conforme Lima LG, et al. (2016) é que durante uma pesquisa realizada com as mães ou responsáveis de crianças, foi identificado que alguns profissionais de saúde não orientam as mães a respeito da importância da Caderneta de Saúde da Criança. Com a ausência de orientação torna-se mais frequente o esquecimento e a perda da caderneta, demonstrando, mais um motivo que pode ocasionar um mal preenchimento. Além disso, ocorre também o desinteresse e o desconhecimento dos pais e acompanhantes a respeito da sua da sua utilização, conteúdo e informações que a CSC traz, pois eles identificam a caderneta apenas como um simples cartão de vacina. Portanto, as orientações a respeito da caderneta é um exercício importante para a promoção da saúde, tendo em vista que o profissional é capaz de incentivar e estimular os familiares a participar acompanhamento do seu filho, levando em consideração que o estímulo familiar é essencial para o crescimento e desenvolvimento da criança.

\section{CONSIDERAÇÕES FINAIS}

Por fim, conforme a realização do estudo evidenciou-se a importância do instrumento de vigilância infantil CSC e que com o passar dos anos houve seu intenso aperfeiçoamento, para que assim exercesse seu papel de forma integral a saúde das crianças e norteassem pais, responsáveis e profissionais de saúde para melhores condutas. Logo, concluiu-se nas literaturas os principais fatores que interferem para o não preenchimento da CSC foram: a falta capacitação dos profissionais de saúde, sobrecarga de trabalho, má comunicação entre a equipe multiprofissional, falta de suporte e mantimentos nas unidades de saúde, desmerecimento e falta de conhecimento da caderneta pelas mães ou responsáveis pela criança.

\section{REFERÊNCIAS}

1. ABUD SM, GAÍVA MAM. Registro dos dados de crescimento e desenvolvimento na caderneta de saúde da criança. Rev Gaúcha Enferm., 2015; 36(2):97-105.

2. ABREU TGT, et al. Desafios na utilização da caderneta de saúde da criança: Entre real e o ideal. J Manag Prim Health care, 2012; 3 (2): 80-3.

3. ANDRADE GN, et al. Caderneta de Saúde da Criança: experiências dos profissionais da atenção primária à saúde. Revista da Escola de Enfermagem da USP, 2014; 48(5): 857-864.

4. AMORIM LP, et al. Preenchimento da Caderneta de Saúde da Criança nos serviços de saúde em Belo Horizonte, Minas Gerais, Brasil. Epidemiol. Serv. Saúde, 2018; 27(1): e201701116.

5. BRASIL. Ministério da Saúde (MS). Secretaria de Atenção em Saúde. Departamento de Ações Programáticas. Política Nacional de Atenção Integral à Saúde da Criança: orientações para implementação Brasília: MS; 2018. 
6. BRASIL. Ministério da Saúde. Saúde da criança: crescimento e desenvolvimento, Secretaria de Atenção à Saúde. Departamento de Atenção Básica. Brasília: Ministério da Saúde, 2012; 33: 272.

7. CAVALCANTE JRC, et al. Percepção das mães sobre o cuidado à criança por meio da caderneta de saúde. Rev. Itinerarius Reflectionis, 2018.

8. COSTA JSD, et al. Assistência à criança: preenchimento da caderneta de saúde em municípios do semiárido brasileiro. Rev. Bras. Saúde Matern. Infant., 2014; 14 (3): 219-227.

9. FREITAS JLG, et al. Preenchimento da caderneta de saúde da criança na primeira infância. Rev Bras Promoç Saúde, 2019; $32: 8407$

10. LIMA LG, et al. A utilização da caderneta de saúde da criança no acompanhamento infantil. Rev. Brasileira de ciências da saúde, 2016; 20(2): 167-174.

11. MENDES KDS, et al. Revisão integrativa: metodo de pesquisa para a incorporação de evidências na saúde e na enfermagem. Texto contexto - enferm., 2008; 17(4): 758-764.

12. PALOMBO CNT, et al. Uso e preenchimento da caderneta de saúde da criança com foco no crescimento e desenvolvimento. Rev Esc Enferm USP, 2014; 48 (Esp): 60-7.

13. REICHERT APS, et al. Vigilância do crescimento e desenvolvimento: análise dos registros na caderneta de saúde da criança. Revista Cogitare Enferm.2016; 21(4): 01-09.

14. ROSOLEM LH, et al. Caderneta de saúde da criança: coordenação do cuidado e acesso à saúde. Revista Cogitare enferm., 2019; 24: e61496.

15. SALLES IC, TORIYAMA, ATM. A Utilização da Caderneta de Saúde da Criança por Alunos de Enfermagem. Revista De Graduação USP, 2017; 2(2): 41-46.

16. SILVA GS, et al. Avaliação da assistência à saúde da criança na Atenção Primária no Brasil: revisão sistemática de métodos e resultados. Revista Ciênc. saúde coletiva, 2020; 25 (8).

17. SILVA FB, GAIVA MAM. Dificuldades enfrentadas pelos profissionais na utilização da caderneta de saúde da criança. Rev. Bras. Pesq. Saúde, 2016; 18(2): 96-103.

18. SILVA TCT, et al. Caderneta de saúde da criança: vigilância do crescimento e desenvolvimento infantil. Rev enferm UFPE online., 2018; 12(12): 3445-55.

19. SOARES JSD, et al. Assistência à criança: preenchimento da caderneta de saúde em municípios do semiárido brasileiro Rev. Bras. Saude Mater. Infant., 2014;14(3).

20. SOUZA NS, et al. Vigilância e estímulo do crescimento e desenvolvimento infantil. Rev enferm UFPE on line., 2019; 13(3): 680-9. 